\title{
Magnons Dampen Skyrmion Motion
}

\section{Adding a friction term to models helps them better account for how spin textures evolve experimentally at room temperature.}

\author{
By Rachel Berkowitz
}

$\mathrm{M}$ odels of spin systems typically treat vortex-like spin configurations called skyrmions as quasiparticles whose motion follows a gyroscopic pattern. But the predictions of the equation currently used to describe such motion-Thiele's equation-don't match up with experimental observations of how skyrmions and other spin textures move. Markus Weißenhofer and his colleagues at the University of Konstanz in Germany now resolve this issue by adding an additional friction term to Thiele's equation.

Thiele's equation was established in the 1970s as the equation of motion for single skyrmionic quasiparticles. It successfully describes both current-driven motions and Brownian motions of skyrmions at low temperatures and high damping. The equation fails, however, at high temperatures and low damping.

In their simulations, Weißenhofer and his colleagues performed numerical simulations to explore the influence of spin damping in a metallic thin film. They found that at finite temperatures,

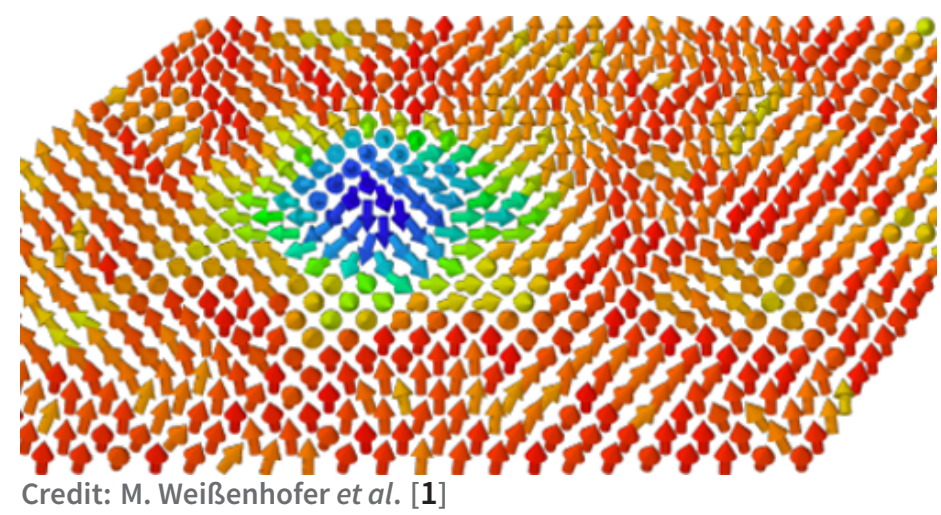

spin waves called magnons appear, which damp the motion of skyrmions already present in the film. Adding a friction term to Thiele's equation to account for this damping, the team found that their updated equation of motion successfully described experimental results. The magnitude of the new term increases linearly with temperature and is independent both of other forms of damping and of skyrmion size.

The researchers say that their result provides a simple framework in which to better predict the dynamics of spin textures. This framework could be useful to experimentalists as they incorporate skyrmions into spintronic technologies, such as energy storage devices.

Rachel Berkowitz is a Corresponding Editor for Physics based in Vancouver, Canada.

\section{REFERENCES}

1. M. Weißenhofer et al., "Skyrmion dynamics at finite temperatures: Beyond Thiele's equation," Phys. Rev. Lett. 127, 047203 (2021). 